\title{
Analysis of Liquid Crystal Tunable Thin-Film Optical Filters Using Signal Flow Graph Technique
}

\author{
Abedalgany Athamneh (iD) ${ }^{1}$ and Shadi A. Alboon ${ }^{1,2}$ \\ ${ }^{1}$ Hijjawi Faculty for Engineering Technology, Yarmouk University, Irbid, Jordan \\ ${ }^{2}$ College of Engineering and Technology, American University of the Middle East, Egaila, Kuwait
}

Correspondence should be addressed to Abedalgany Athamneh; athamneh@yu.edu.jo

Received 22 February 2021; Accepted 29 June 2021; Published 13 July 2021

Academic Editor: Mustapha Tlidi

Copyright (C) 2021 Abedalgany Athamneh and Shadi A. Alboon. This is an open access article distributed under the Creative Commons Attribution License, which permits unrestricted use, distribution, and reproduction in any medium, provided the original work is properly cited.

In this paper, a liquid crystal tunable thin-film optical bandpass filter is studied and analyzed using the signal flow graph technique. This paper investigates an exact form for calculating the transmission coefficients, reflection coefficients, and the transmission intensity of the filter. The simulation results show the filter performance and the channel shape profile. In addition, the results show the tuning capability of the filter. The signal flow graph technique provides an attractive method for analyzing the thin-film optical filters since it overcomes the difficulty of the refractive index concept in extending to optical applications. Moreover, it simplifies the filter analysis and design process.

\section{Introduction}

Optical filters are considered as one of the most important elements of optical and electro-optical systems technology. Laser, photodetectors, and optical filters represent some of the devices used in the optical communication networks. The tunable optical filters are considered vital components in the huge volume optical communication systems based on the wavelength multiplexing approach $[1,2]$. The performance of the optical filters (bandwidth, slope, insertion loss, and flatness) affects the overall signal to noise ratio (SNR) and the bit error ratio (BER) of optical communication systems. Therefore, effective analysis methods and approaches are required to analyze the optical filter.

Several methods have been proposed for the analysis of optical filters. Some of these methods are the matrix method, the transfer matrix/chain matrix algebraic method, and the method of solving the field equations.
These methods are hard to be extended to optical applications because of the dependency in optics on the concepts of refractive index [3]. Signal flow graph has been proposed to overcome the drawbacks of the other approaches in difficulties due to the dependence in optics on the concepts of refractive index and to avoid the repeated summations in some other methods. Besides, it simplifies the filter analysis, and it can be used in the analysis and design of both the optical and the electrical systems. In [3], a multicomponent cavity system in the laser has been analyzed using the signal flow graph technique, and the author has proved that it is a rapid and convenient method in analyzing the cavities involving active components. A microwave approach and signal flow graph (SFG) approach were applied to analyze an optical multilayer system in [4]. It was shown that the signal flow graph approach is a powerful approach, simple, and has visual advantages for design. A signal flow graph structure of the high pass filter useful for 
operational transconductance amplifier and capacitor (OTA-C) has been presented in [5]. A fiber optical resonating structure has been designed and analyzed using the SFG technique in [6]. Also, a comparison of various analytical techniques has been made in order to highlight the typical advantage of using the SFG.

\section{Signal Flow Graph}

A signal flow graph technique is used in order to generate a graphical description of a vibrant system of linear equations. The SFG contains several nodes which embrace the data momentarily and pathways to pass on the data among those nodes [7]. Each pathway is guided from the origin to the result, and it is characterized by a factor named the gain transmittance of the pathway. Moreover, the signal flow graph can be redefined in terms of paths and loops. The loop is a path that starts and finishes at the same node with no node passed by the loop more than once. The loops of SFG are also characterized by the order of each loop. A first-order loop consists of single loop, while a second-order loop consists of two nonoverlapping single loops [8]. A loop contains many single untouching loops which is called a higher-order loop. The gain transmittance of the second-order and higher order loops can be found by multiplying the gain transmittance of individual first-order loops that create the second-order and the higher order loops [8]. The gain transmittance of the flow graph between a given input and output nodes can be evaluated by Mason's formula:

$$
T=\frac{\sum P_{n}\left[1-\sum L_{n}(1)+\sum L_{n}(2)-\cdots+(-1)^{i} \sum L_{n}(i)+\cdots\right]}{1-\sum L(1)+\sum L(2)-\cdots+(-1)^{i} \sum L(i)},
$$

where $P_{n}$ is the coefficient of the $n^{\text {th }}$ pathway, $\sum L_{n}(i)$ is the summation of the coefficients of all the $i^{\text {th }}$-order loops that do not touch the $n^{\text {th }}$ pathway $P_{n}$, and $\sum L(i)$ is the summation of the coefficients of all the $i^{\text {th }}$-order loops in the flow graph. In order to analyze a multilayer optical filter using Mason's rule and SFG theory, we will apply this theory to a simpler multilayer filter analysis. After that, we will apply it to a more complex multilayer filter analysis. A three-layer optical structure will be analyzed first. To begin analysis, we will assume that all layers are lossless, the optical wave incident to the threelayer system polarization is a transverse electric (TE) polarization. As the LC used in this study is (BL006) which is sensitive to TE polarization only, the alignment director is parallel to TE polarization [9]. Moreover, the incidence of the TE wave at the boundaries of the different layers is normal. The signal flow graph representation is shown in Figure 1. To find the transmittance of the three-layer optical system, there is one pathway (abcdef) and one loop (cdlm). Applying Mason's rule to Figure 2, for input $a$ to the output at node $f$, we have

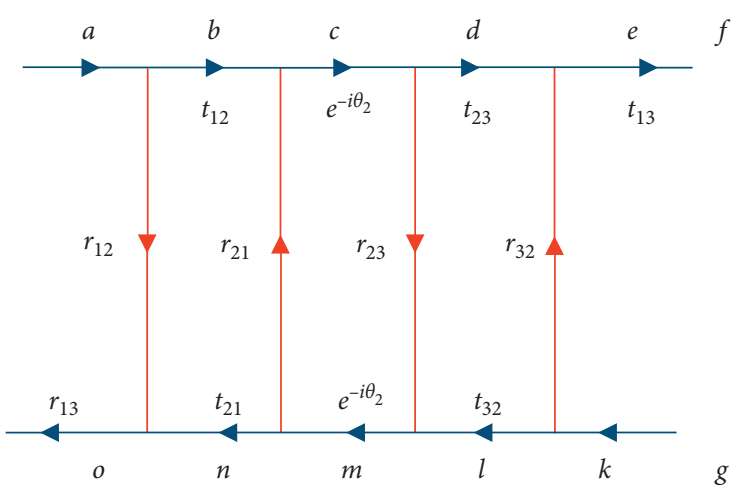

FIGURE 1: SFG illustration for a three-layer optical system.

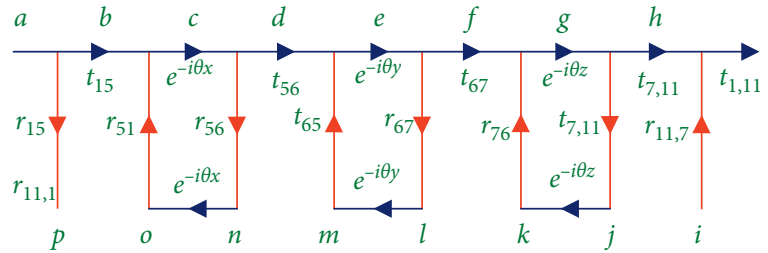

FIGURE 2: SFG representation for the optical filter after using single path transformation.

$$
\begin{aligned}
P_{1} & =t_{12} t_{23} e^{-i \theta_{2}}, \\
\Delta & =1-r_{21} r_{23} e^{-2 i \theta_{2}}, \\
\Delta_{1} & =1 .
\end{aligned}
$$

From equations (1)-(4), the transmission coefficient $t_{13}$ at node $f$ is

$$
t_{13}=\frac{t_{12} t_{23} e^{-i \theta_{2}}}{1-r_{21} r_{23} e^{-2 i \theta_{2}}} .
$$

Now, the intensity transmittance of the path from layer 1 to layer 3 can be determined using the following equation:

$$
T_{13}=\frac{n_{3}}{n_{1}} t_{13} t_{13}^{*}
$$

where $n_{1}$ and $n_{3}$ are the refractive indices for the first and third layer, respectively. Using the same procedure to find the reflectivity, then we get

$$
\begin{aligned}
& r_{13}=\frac{r_{12}+r_{23} e^{-2 i \theta_{2}}}{1-r_{21} r_{23} e^{-2 i \theta_{2}}}, \\
& r_{31}=\frac{r_{32}+r_{21} e^{-2 i \theta_{2}}}{1-r_{21} r_{23} e^{-2 i \theta_{2}}}, \\
& t_{31}=\frac{t_{32} t_{21} e^{-i \theta_{2}}}{1-r_{21} r_{23} e^{-2 i \theta_{2}}} .
\end{aligned}
$$

In the five-layer optical system, the analysis will depend on the results previously attained from the analysis of the 
three-layer system, because we can implement this system as a cascade of two three-layer system. Since the transmission coefficients $t_{13}$ and $t_{31}$ have been derived in equations, they can be used in this structure analysis, which helps to reduce the number of anticipated branches of the SFG. Then, the analysis results will be as follows:

$$
\begin{aligned}
& t_{15}=\frac{t_{13} t_{35} e^{-2 i \theta_{3}}}{1-r_{31} r_{35} e^{-2 i \theta_{3}},} \\
& r_{35}=\frac{r_{34}+r_{45} e^{-2 i \theta_{4}}}{1-r_{34} r_{45} e^{-2 i \theta_{4}}} .
\end{aligned}
$$

Now, the intensity transmittance of the pathway from layer 1 to layer 3 can be determined using the following equations:

$$
\begin{aligned}
& T_{15}=\frac{n_{5}}{n_{1}} t_{15} t_{15}^{*}, \\
& r_{15}=\frac{r_{13}+r_{35} e^{-2 i \theta_{3}}}{1-r_{31} r_{35} e^{-2 i \theta_{3}},} \\
& r_{51}=\frac{r_{53}+r_{31} e^{-2 i \theta_{3}}}{1-r_{31} r_{35} e^{-2 i \theta_{3}} .}
\end{aligned}
$$

\section{Liquid Crystal Tunable Optical Filter Structure}

The structure of the LC tunable filter is composed of two cascaded five-layer optical structure (with alternating high and low refractive index materials) to form the reflective mirrors, with an LC sandwiched in between. The tuning capability in the suggested filter design is realized by inserting a liquid crystal (LC) in the filter cavity as the tuning medium. The effective refractive index of the nematic LC layers can be controlled applying an electric field. Application of the electric field will change the LC molecules orientation which results in changing the LC layer refractive index to obtain the required tuning capability [9]. Based on this structure, we can use the previous signal flow graph analysis results for such a filter. The signal flow graph representation for the filter is shown in Figure 3. The transmission and the reflectivity coefficients can be calculated using the same procedure discussed previously for the three- and five-layer structures.

In order to derive the transmission coefficient and reflectivity, we follow the same procedure as before. The signal flow graph implementation of Figure 3 is transformed using a single path transformation method which was discussed by Lee and Whitaker [6], where the reflective paths are excluded. The resulted new topographical transform is shown in Figure 2.

\section{Results and Discussion}

The filter design shown in Figure 3 has been simulated using the SFG analysis results derived in Section 3. The filter design

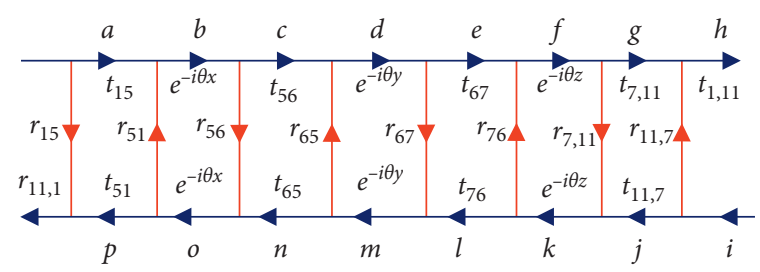

FIgURE 3: Reduced SFG representation for the optical filter.

parameters used were as follows: the high and low refractive index materials were silicon $(\mathrm{Si})$ and silicon dioxide $\left(\mathrm{SiO}_{2}\right)$, respectively. These alternative layers are used to form the reflective layers. The liquid crystal used was BL006 which has a refractive index tuning range of $n=1.5-1.72$. The results for the transmission profile are shown in Figures 4 and 5.

Figures 4 and 5 show the channel shape profile for the LC refractive index $(n=1.5$ and $n=1.72)$, respectively. The transmission profile has a sharp flat-top response with ripple less than $0.1 \mathrm{~dB}$ for both figures. As seen from Figures 4 and 5 , this filter has a tuning range around $170 \mathrm{~nm}$. Moreover, this tuning range covers the $C$ and the $L$ bands.

The profile width for typical bandwidths: $-3 \mathrm{~dB}$ and $-0.5 \mathrm{~dB}$ are given on both figures. It is noteworthy to say that the values of these bandwidths are different for the two figures. This is due to the fact that the chromatic dispersion of the filter, i.e., the dependence of the refractive index on the wavelength, which leads to widening the bandwidth of the filter [10]. Even though the widths are almost doubled for the -3 and $-0.5 \mathrm{~dB}$ bandwidths, we still have $\mathrm{a}-3 \mathrm{~dB}$ bandwidth less than $2 \mathrm{~nm}$ that is the standard for such filters. The dispersion factor was considered in the values of the LC refractive index by using the following equation [11]:

$$
n(\lambda)=A+\frac{B}{\lambda^{2}}+\frac{C}{\lambda^{4}}
$$

where $A, B$, and $C$ are constants that can be found in (9).

Figure 6 shows the filter channel shape for different values of the LC refractive index. We can see from the figure that the filter has the tuning capability, as for each value of LC refractive index, we have different center frequency for the filter transmission profile. The refractive index values used in this figure are close to each other in order to make the figure clearer. Such small value change required a stable and accurate voltage source [9].

\section{Conclusions}

Tunable optical filters are widely needed in optical communication systems. This paper illustrates the analysis of liquid crystal tunable optical thin film filters using the SFG technique. A detailed derivation of the SFG analysis is presented in this paper. The paper provides an exact form for calculating the transmission coefficients, reflection coefficients, and the transmission intensity. The resulted equations described the transmittance of eleven-layer thin film optical filters with a LC crystal inserted between the reflective layers. The simulation results show that the filter has a flat-top sharp response. Moreover, the results show the 


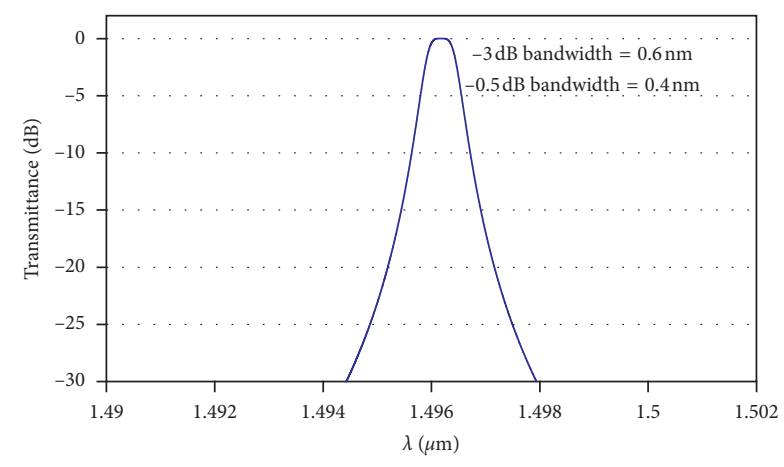

FIgURE 4: The channel shape profile for the LC refractive index $(n=1.5)$.

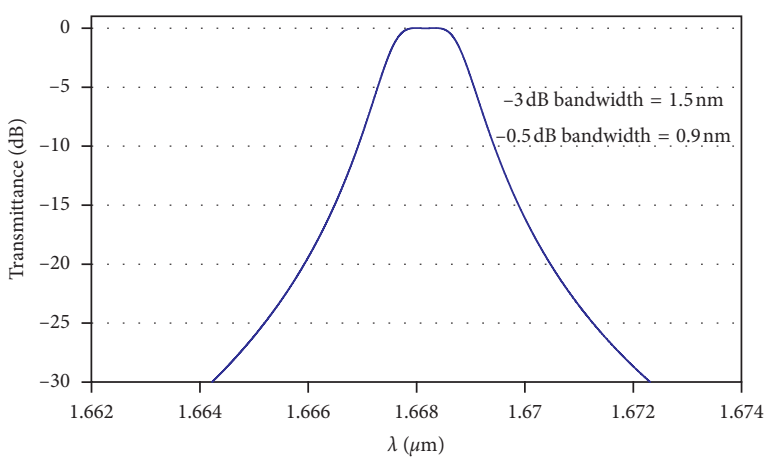

FIGURE 5: The channel shape profile for the LC refractive index $(n=1.72)$.

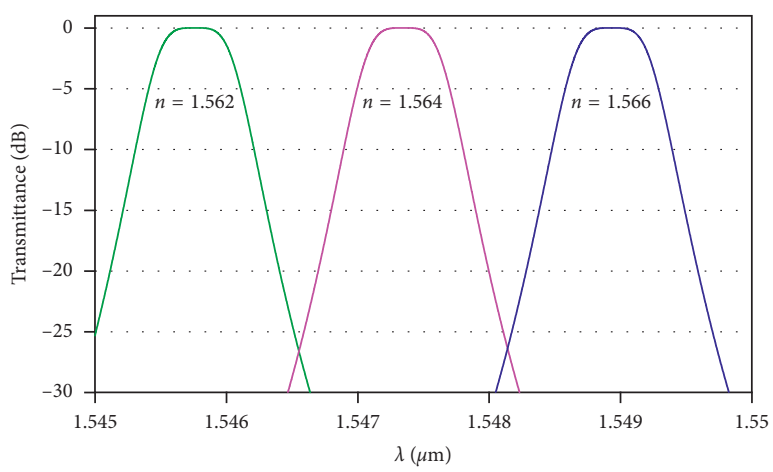

FIgURE 6: The transmission intensity for different values of the LC refractive index.

tuning capability of the filter. The SFG technique provides a simple and accurate analysis method for the tunable thin film optical filters.

\section{Data Availability}

No data were used to support this study.

\section{Conflicts of Interest}

The authors declare that they have no conflicts of interest.

\section{References}

[1] S. A. Alboon and R. G. Lindquist, "Flat top liquid crystal tunable filter using coupled Fabry-Perot cavities," Optics Express, vol. 16, no. 1, pp. 231-236, 2008.

[2] S. A. Alboon and R. G. Lindquist, "Flat-top/distortionless tunable filters based on liquid crystal multi cavities for DWDM applications," in Proceedings of the IEEE Southeastcon, pp. 117-122, Huntsville, AL, USA, April 2008.

[3] M. H. Dunn, "Use of flow graphs in the analysis of optical cavities," Applied Optics, vol. 10, no. 6, pp. 1393-1397, 1971.

[4] D. Lee and J. F. Whitaker, "Analysis of optical and terahertz multilayer systems using microwave and feedback theory," Microwave and Optical Technology Letters, vol. 51, no. 5, 2009.

[5] M. Guzinski, "Signal flow graph structure of high-pass filter useful for OTA-C," in Proceedings of the IEEE 39th Midwest Symposium on Circuits and Systems, vol. 2, pp. 929-932, Estes Park, CO. USA, April 1997.

[6] S. Srivastava and K. Srinivasan, "Signal flow graphs in the analysis and design of fiber optical resonating structures," in Proceedings of Photonic, Monterey, CA, USA, November 2004.

[7] I. J. Nagrath, Control Systems Engineering, New Age International, Chennai, India, 2006.

[8] U. A. Bakshi and S. C. Goyal, Feedback Control Systems, Technical Publications, Chennai, India, 2009.

[9] S. A. Alboon, A. S. Abu-Abed, R. G. Lindquist, and H. R. AlZoubi, "Novel liquid crystal tunable flat-top optical interleaver," Progress In Electromagnetics Research B, vol. 19, pp. 263-283, 2010.

[10] S. Zhu, M. Li, N. H. Zhu, and W. Li, "Chromatic-dispersioninduced power-fading suppression technique for bandwidthquadrupling dual-chirp microwave signals over fiber transmission," Optics Letters, vol. 44, no. 4, pp. 923-926, 2019.

[11] P. García Parejo and A. Álvarez-Herrero, "Liquid crystals for space instrumentation: optical properties of liquid crystal mixtures for polarimeters," Optical Material Express, vol. 9, pp. 2681-2698, 2019. 